\title{
Psychosoziale und berufliche Folgen der Depression
}

\author{
A. Lang', J. Hamann², P. Brieger ${ }^{1}$ \\ ${ }^{1}$ kbo-Isar-Amper-Klinikum München-Ost, Klinik; ${ }^{2}$ Klinik und Poliklinik für Psychiatrie und Psychotherapie, TU München; \\ Arbeitsgruppe "Return-to-Work" München (gefördert durch den Innovationsfond des GBA Förderkennzeichen \\ 01VSF17012)
}

\author{
Schlüsselwörter \\ Depression, Krankheitslast, berufliche Reha- \\ bilitation
}

Depressionen gehören zu den häufigsten Formen psychischer Erkrankungen in unserer Gesellschaft und gehen mit einer hohen Krankheitslast einher. Für die Betroffenen führt dies zu Beeinträchtigungen in allen Aspekten ihrer Lebensführung. Auf psychosozialer Ebene äußert sich dies $u$. a. in familiären Schwierigkeiten sowie einer Verarmung der sozialen Situation. Auf beruflicher Ebene kommt es zu Einbußen in der Leistungsfähigkeit bis hin zur Arbeitsunfähigkeit und Frühverrentung. Die Wiederherstellung der psychosozialen sowie beruflichen Leistungsfähigkeit gilt daher als wichtiges Ziel der Behandlung. Den psychosozialen Beeinträchtigungen kann mit unterschiedlichen psychotherapeutischen Interventionen entgegengewirkt werden. Der Klärung der beruflichen Perspektiven und der Rückkehr an den Arbeitsplatz wird jedoch in der Versorgung noch zu wenig Beachtung geschenkt. Dies sollte stärker in den Fokus gesetzt werden, um die verschiedenen Möglichkeiten zur Unterstützung der beruflichen Wiedereingliederung ausschöpfen zu können.

\section{Zusammenfassung}

\section{Keywords}

Depression, burden of illness, vocational rehabilitation

\section{Summary}

Depression is one of the most common mental disorders in our society and comes along with a high burden of disease. This leads to serious impact on the lifestyle of those affected. On the psychosocial level this manifests itself, among other things, in family difficulties as well as in the impoverishment of the social situation. At the professional level depression leads to a loss of performance up to an inability to work and early retirement. The restoration of the psychosocial and occupational capacity is therefore an important treatment goal. With various psychotherapeutic interventions the psychosocial impairment can be counteracted. However, still little attention is given to the clarification of vocational opportunities and a return to work. There must be a stronger focus on this topic so that the various possibilities of support for vocational reintegration can be exploited.

Korrespondenzadresse

Prof. Dr. Peter Brieger

kbo-Isar-Amper-Klinikum

Vockestr. 72,85540 Haar bei München

Tel. 089/45623203, Fax 089/45623199

peter.brieger@kbo.de
Psychosocial and professional consequences of depression

Nervenheilkunde 2018; 37: 575-580

eingegangen am: 10. Juni 2018

angenommen am: 28. Juni 2018
„Reiß dich mal zusammen!“ Solche und ähnliche Ratschläge erhalten depressive Menschen ständig von ihrem privaten und beruflichen Umfeld. Durch diese gut gemeinten Ratschläge fühlen sich Patienten nicht ernst genommen. Obwohl eine Ent- stigmatisierung langsam Einzug hält, ist dennoch ein umfassendes Verständnis noch nicht angekommen. Die Stiftung Deutsche Depressionshilfe hat mit dem Deutschland-Barometer Depression 2017 eine repräsentative Befragung zu den An- sichten und Einstellungen der Bevölkerung zur Depression erhoben (1). Sehr häufig wird sie als Reaktion auf widrige Lebensumstände verstanden und nicht als eigenständige und schwere Erkrankung erkannt. Bei 30\% der Befragten hält sich das Vorurteil, Depression sei ein Zeichen von Charakterschwäche oder Folge einer falschen Lebensführung. Gefragt nach Behandlungsmöglichkeiten gab jeder fünfte Befragte an, Süßigkeiten oder Sich Zusammenreißen würde bei der Bewältigung helfen.

Auch das bisherige Verständnis der Depression als vergleichsweise gut behandelbare Erkrankung, mit hohen Remissionsraten und keine nachweisbaren organischen Folgeschäden hält nach heutigem Wissensstand nicht (2). Es handelt sich um eine Erkrankung mit hoher Krankheitslast für den Betroffenen, die viel häufiger als früher angenommen auch einen chronischen Verlauf nehmen kann.

\section{Epidemiologie}

Laut der Studie zur Gesundheit Erwachsener in Deutschland (DEGS) sowie Studien der Organisation für wirtschaftliche $\mathrm{Zu}$ sammenarbeit und Entwicklung (OECD) erkranken 43\% der Bevölkerung irgendwann in ihrem Leben an einer psychischen Störung (3). Für diagnostizierte Depressionen liegt die 12-Monatsprävalenz bei Erwachsenen im Alter von 18-65 Jahren in Deutschland bei 11\% (4). Betrachtet man die Lebenszeitprävalenz liegt diese mit 19\% deutlich höher. Depressionen gehören somit zu den häufigsten Formen psychischer Erkrankungen. Seit Beginn der 1990er-Jahre sind Depressionen als die gesellschaftlich belastendste Krankheitsgruppe einzuordnen (5). 
Nach Schätzungen der Global Burden of Disease Studie der WHO sind Depressionen weltweit eine Hauptursache für krankheitsbedingte Behinderung $(6,7)$. Mit der Messgröße YLD (years of life with disability) erfasst das Konzept der Global Burden of Disease, wie viele Lebensjahre mit Behinderung gelebt werden. Weltweit haben Depressionen 11,8\% der YLD verursacht. Dies entspricht 67 Mio. durch Behinderung beeinträchtigte Lebensjahre (8).

Depressionen führen zu umfangreichen Krankheitsfolgen, sowohl auf Ebene des Individuums als auch auf Ebene der Gesellschaft. Aufgrund ihrer Erkrankung sind depressive Menschen meist in allen Aspekten ihrer Lebensführung beeinträchtigt. Personen mit aktuellen psychischen Störungen berichten dreimal so häufig von Einschränkungstagen im Vergleich mit Personen, die niemals eine psychische Störung hatten (9).

Ergebnisse einer Studie der Weltgesundheitsorganisation in deutschen Allgemeinarztpraxen zur Chronifizierung und psychosozialen Behinderung bei depressiven Patienten zeigen, dass jeder dritte bis vierte Patient sowohl in der objektiven als auch in der subjektiven Beurteilung deutlich ausgeprägte Behinderung im Vollzug seiner alltäglichen Rollen zeigt (10). 50\% der Patienten mit einer ausgeprägten depressiven Episode erleben Beeinträchtigungen in ihrer Funktionsfähigkeit, die zu Schwierigkeiten in der Ausführung ihrer Alltagsrollen führen (11). Sie fühlen sich in ihrer Lebensqualität sowie in ihrer Fähigkeit zur Auseinandersetzung mit Lebensanforderungen beeinträchtigt (10).

Die Symptome der Depression verursachen bei den meisten Erkrankten starke Beeinträchtigungen sowohl der körperlichen als auch der psychischen Verfassung. Die Hauptsymptome einer depressiven Episode sind nach ICD-10 (12) depressive, gedrückte Stimmung, Interessenverlust und Freudlosigkeit sowie die Verminderung des Antriebs, die mit erhöhter Ermüdbarkeit und Aktivitätseinschränkung selbst nach kleinen Anstrengungen einhergeht.

Nachfolgende Übersicht betrachtet die daraus erwachsenden psychosozialen und insbesondere beruflichen Folgen depressiver Erkrankungen.

\section{Psychosoziale Folgen}

\section{Zwischenmenschliche Beziehungen}

Kaum eine psychische Störung verursacht solch massive psychosoziale Beeinträchtigungen wie die unipolare Depression (13). Durch die depressive Symptomatik leiden unter anderem die sozialen Beziehungen der Erkrankten. Unterschiedliche Studien haben belegt, dass depressive Personen ihre sozialen Kontakte als wenig unterstützend und erfüllend sowie zum Teil belastend erleben $(9,14)$. Die negativen Denkmuster depressiver Personen schlagen sich zudem auf die Gestaltung ihrer zwischenmenschlichen Beziehungen nieder. So sind die Reaktionen des Umfelds häufig durch Ablehnung und Zurückweisung gekennzeichnet (14).

Nicht nur die Erkrankten selbst leiden unter den zahlreichen Beeinträchtigungen, die mit der Erkrankung einhergehen. Auch die Partner sowie Familienangehörige werden vor hohe Anforderungen gestellt, wodurch die familiäre und Partnerbeziehung häufig in Mitleidenschaft gezogen werden (14-16). Die veränderten Bedürfnisse des Erkrankten erfordern erhöhte Rücksichtnahme sowie zum Teil sogar eine Veränderung des gesamten Tagesablaufs. Diese $\mathrm{Zu}$ nahme an Belastungen und Änderungen kann schnell zu Konflikten führen (17).

In Deutschland leben ca. 500000 Kinder mit einem depressiven Elternteil (2). Die Auswirkungen auf die betroffenen Kinder, wie ein verringertes Selbstwertgefühl sowie Verhaltensauffälligkeiten wurden in Studien deutlich $(15,18)$. Kinder depressiver Eltern fällt es zum Teil selbst schwer, Freude zu empfinden. Das Risiko eines Kindes depressiver Eltern selbst an einer depressiven Störung zu erkranken, ist um das ca. Sechsfache erhöht (17). Auch die Parentifizierung ist zu beobachten. Hier übernehmen die Kinder die Eltern- bzw. Partnerfunktion. In den meisten Fällen können sie jedoch den Anforderungen dieser Rolle kaum gerecht werden. Ihre eigenen Bedürfnisse werden hinter die der Eltern gestellt, was wiederum negative Auswirkungen auf ihre eigene Entwicklung hat (17).

Auch die Erziehungskompetenz der Eltern nimmt bei der Entwicklung der Kinder eine entscheidende Rolle ein. Diese ist jedoch bei depressiv Erkrankten zum Teil stark beeinträchtigt (17). In der Studie von Zimmermann et al. konnte gezeigt werden, dass vor allem mehrfacherkrankte Depressive eine höhere objektive Belastung in der Kindererziehung erleben sowie auch mehr Schwierigkeiten im Umgang mit ihren Kindern aufweisen (19). Gefragt nach ihren eigenen Empfindungen, berichten Patienten von dem Wunsch, wieder eine normale Beziehung mit Freunden und Familie führen zu können und gute Eltern für ihre Kinder zu sein (20).

\section{Einschränkung des Aktivitäts- niveaus}

Durch die Weissenauer Katamensestudie konnte vor allem auch eine Auswirkung der depressiven Symptomatik auf die Freizeitaktivität der Erkrankten gezeigt werden (9). Im Vergleich zur Normalbevölkerung weisen sie ungünstigere Bedingungen im Bereich der Freizeitaktivitäten auf. Objektiv bestehen die gleichen Möglichkeiten der Freizeitgestaltung, die jedoch von depressiven Personen schlechter genutzt werden. Vor allem durch die viele Zeit, die zu Hause ohne Tätigkeit verbracht wird, kommt es zu einer Verarmung der sozialen Situation, die sich in der Einschränkung des Aktivitätsniveaus sowie der Sozialkontakte zeigt.

Stellt sich eine Verbesserung der Symptomatik ein, kommt es auch zu einer Verbesserung der sozialen Situation. Bei chronischem Verlauf der Erkrankung ist jedoch mit einer ungünstigeren Entwicklung der psychosozialen Bedingungen zu rechnen (9). Die Zunahme des Schweregrades der Symptome geht mit einer Erhöhung der psychosozialen Beeinträchtigungen einher (13).

\section{Berufliche Folgen}

\section{Arbeitsunfähigkeit}

In den ökonomisch entwickelten Ländern nimmt die Arbeitsunfähigkeit aufgrund psychischer Störungen beständig zu (21). Beschäftigte mit psychischen Störungen werden verglichen mit anderen Erkrankungen besonders häufig arbeitsunfähig geschrieben. Die Analyse der Daten unterschiedlicher Krankenkassen zeigte auf, dass psychisch erkrankte Arbeitnehmer 2012 ca. 
70 Millionen Tage in ihren Betrieben gefehlt haben (22). 2006 zeichnete sich in den Vereinigten Staaten ein ähnliches Bild. Pro Jahr waren es hier 225 Millionen gefehlte Arbeitstage im Betrieb sowie eine Summe von 36,6 Milliarden US-Dollar durch Produktivitätsverluste (23).

Die erheblichen Einbußen an Lebensqualität gehen auch mit Beeinträchtigungen der Leistungsfähigkeit einher (3). Depressive Symptome wie Einschränkungen der Anpassungs- und Konzentrationsfähigkeit, sozialer Rückzug und vermindertes Selbstwertgefühl gefährden die Arbeitsfähigkeit $(3,15)$. Studien haben gezeigt, dass depressiv erkrankte Arbeitnehmer im Vergleich $\mathrm{zu}$ einer gesunden Kontrollgruppe eine deutlich herabgesetzte Leistungsfähigkeit zeigen (21). Dies konnte vor allem in den Punkten interpersonelle Aufgabenbereiche, Fertigstellung der Arbeitsaufträge, Zeitmanagement und der körperlichen Bewältigung von Arbeit gesehen werden. Laut einer Studie im Dienstleistungssektor entspricht die reduzierte Arbeitsleistung nicht krankgeschriebener Depressiver pro Monat 2,3 Abwesenheitstage $(24,25)$. In akuten depressiven Phasen kann die Leistungsfähigkeit so gravierend eingeschränkt sein, dass dies zur Arbeitsunfähigkeit führt (5).

Laut Fehlzeitenreport 2017 der AOK waren 11\% der bundesweiten Fehlzeiten auf psychische Erkrankungen zurückzuführen. Die durchschnittliche Falldauer psychischer Erkrankungen ist mit 25,7 Tagen je Fall mehr als doppelt so lange wie der Durchschnitt (26). Die durch Depression bedingten Arbeitsunfähigkeitstage haben in Deutschland kontinuierlich zugenommen $(4,5,27)$. Nach Angaben der Deutschen Angestellten Krankenkasse waren depressive Episoden im Jahr 2013 unter den psychischen Störungen die häufigste Einzeldiagnose im Zusammenhang mit Arbeitsausfalltagen (15). In Deutschland haben Arbeitnehmer, die an einer depressiven Störung leiden, mit 19,8 Tagen ca. doppelt so viele Fehltage wie Nichtdepressive. Seit 1994 ist ein Anstieg der Arbeitsunfähigkeitsfälle um 120\% zu verzeichnen (3). Der Anstieg der Fehltage aufgrund psychischer Erkrankungen ist eine der auffälligsten Entwicklungen in Bezug auf die Krankenstandskennziffern in den letzten Jahren. Auch 2017 setzte sich die Zahl der
Krankschreibungsfälle aufgrund psychischer Erkrankungen fort, wobei die weitaus meisten Fehltage durch Depressionen verursacht wurden (28).

\section{Frühberentung}

Depressionen führen pro Jahr neben ca. 11 Millionen Arbeitsunfähigkeitstagen zudem zu rund 15000 Frühberentungen (8). Depressive Erkrankungen sind weltweit eine der führenden Gründe für Behinderung und Frühberentung (21). Auch bezüglich der Erwerbsunfähigkeits- und Erwerbsminderungsrente spielt die Diagnose Depression eine entscheidende Rolle. Laut Statistik der Deutschen Rentenversicherung nimmt der Anteil der Erwerbsminderungsrenten wegen psychischer Störungen an der Gesamtheit der Bevölkerung seit $2001 \mathrm{zu}$ (3). Im Jahr 2012 erfolgten 37\% aller Erwerbsminderungsberentungen aufgrund psychischer Störungen. Den größten Anteil am Erwerbsminderungsrentengeschehen der Rentenversicherung machen neben neurotischen, Belastungs- und Anpassungsstörungen psychisch erkrankte Menschen mit affektiven Störungen aus (3). Im Jahr 2008 lagen die verlorenen Erwerbstätigkeitsjahre für alle Erkrankungen bei 4,25 Mio. Davon waren 763000 verlorene Erwerbstätigkeitsjahre durch psychische Störungen verursacht. Fast ein Drittel war auf die Gruppe der Depressionen zurückzuführen (15). Inzwischen geht fast jede zweite Frühberentung (43\%) auf eine psychisch bedingte Erwerbsminderung zurück (26). Im Vergleich zu allen anderen Krankheiten führt keine andere Erkrankung so häufig zu einer vorzeitigen Verrentung wie die unipolare Depression (22).

\section{Zunahme der Prävalenz?}

Trotz eines allgemeinen Rückgangs des Krankenstandes über alle Krankheitsarten hinweg, steigen die Arbeitsunfähigkeit sowie die Erwerbsminderungs- und Erwerbsunfähigkeitsrente aufgrund psychischer Störungen kontinuierlich an. Daher stellt sich bereits seit einigen Jahren die Frage nach der Erhöhung der allgemeinen Prävalenz psychischer Störungen in der Bevölkerung. Sowohl deutsche als auch internatio- nale Untersuchungen konnten zeigen, dass ein hohes Maß an Konstanz der Prävalenzdaten vorliegt (29). Insgesamt ist es in den letzten Dekaden zu keiner bedeutsamen Zunahme psychischer Störungen gekommen (30). Wie lassen sich dann die gestiegenen Arbeitsunfähigkeits- sowie Frühverrentungszahlen aufgrund depressiver Störungen erklären? Im Laufe der letzten Jahre ist es zu einer vermehrten „Normalisierung" psychischer Störungen gekommen. Dies führt zu einer vermehrten Akzeptanz und Aufmerksamkeit psychischer Probleme und deren Symptome. Auf Patientenseite werden mehr psychische Symptome wahrgenommen und dem Arzt berichtet. Diese positive Entwicklung kann jedoch auch dazu führen, dass sich Menschen als zu behandlungsbedürftig sehen. Wie auch bei körperlichen Erkrankungen besteht nicht immer die Notwendigkeit einer unmittelbaren Behandlung (30).

Auch auf Seite der Hausärzte kommt es zu einer zunehmenden Sensibilität in Bezug auf das Thema psychischer Erkrankungen, wodurch diese häufiger als früher erkannt werden. Durch den Zusammenhang somatischer Erkrankungen und Depression kann es jedoch sein, dass Symptome von Ärzten anders zugeordnet werden als in der Vergangenheit. So kann z. B. aufgrund des Zusammenhangs zwischen Rückenschmerzen und Depression ein Diagnosewechsel von unspezifischen Rückenschmerzen hin zur Depression erfolgen (22). Auch Angst und somatoforme Störungen werden häufig als Depression klassifiziert (24).

Diskussionen gibt es, dass die Veränderungen und zunehmenden Belastungen im Arbeitsleben zu einem Zuwachs depressiver Störungen führen. Die Folgen des Wettbewerbsdrucks auf Unternehmen führen $\mathrm{zu}$ Arbeitsintensivierung und erhöhten Lern- und Anpassungserfordernissen bei den Beschäftigten. Zum Teil schwierige Beschäftigungsverhältnisse verursachen $\mathrm{zu}$ dem Arbeitsplatzunsicherheit. Dies führt zu erhöhten psychomentalen und sozioemotionalen Belastungen (31). Von Arbeitnehmern wird mehr Engagement, Flexibilität und kontinuierliche Anpassung an sich ständig ändernde Bedingungen gefordert (32). 


\section{Behandlungsansätze und Interventionen}

Nach den S3-Leitlinien der unipolaren Depression (15) gilt es als allgemeines Behandlungsziel, die berufliche und psychosoziale Leistungsfähigkeit wiederherzustellen. Hierzu kommen in der Regel pharmakologische und/oder psychotherapeutische Ansätze zur Anwendung. Ergänzt werden diese Ansätze häufig mit psychosozialen Interventionen. Mit Blick auf die Behandlung der psychosozialen und beruflichen Folgen der Depression steht die Frage im Mittelpunkt, wie der Betroffene wieder an seinem familiären, beruflichen und gesellschaftlichen Leben teilnehmen kann.

Eine Grundvoraussetzung für die Behandlung aller depressiven Patienten ist die Psychoedukation (33). Es werden dabei verständliche Informationen über depressive Symptome und deren Behandlungsoptionen vermittelt. Somit lassen sich Symptome besser verstehen, der Patient kennt den Verlauf der Erkrankung und wird in seiner Eigenverantwortung gefördert. Für die Angehörigen kann durch die Psychoedukation eine Entlastung entstehen. Es kann zu einer Verbesserung der Interaktion zwischen Patient und Angehörigen sowie $\mathrm{zu}$ einem geförderten gegenseitigen Verständnis kommen.

Den Belastungen auf Angehörigenseite lassen sich zudem durch paar- und familienbezogene Interventionen entgegenwirken (34). Neben der Vermittlung von Wissen über die Erkrankung geht es u. a. um den Abbau von Schuldgefühlen, der Rückbesinnung auf eigene Bedürfnisse, Kommunikations- und Problemlösetraining sowie Strategien der Krisenbewältigung.

Ein weiterer zentraler Bestandteil der Behandlung depressiver Patienten ist die Förderung von Aktivitäten mittels sozialer Fertigkeiten sowie Verhaltensaktivierung (35). Die Förderung sozialer Fertigkeiten befähigt den Patienten darin, seine eigenen Wünsche und Bedürfnisse erkennen und durchsetzen zu können, positive Gefühle zu äußern und soziale Kontakte zu knüpfen sowie Aktivitäten anzugehen. Das kognitive Trainingsmodell der sozialen Fertigkeiten zielt auf die Verbesserung wichtiger kognitiver Fertigkeiten wie z. B. Aufmerksamkeit ab. Mit dem Sozial-kognitivem
Training lassen sich beide Ansätze verknüpfen (36). Mittels der Verhaltensaktivierung lässt sich ein angemessenes Aktivitätsniveau mit einer ausgewogenen Tagesstruktur erreichen. Hierbei gilt es einen Aufbau sowie eine Steigerung positiv erlebter Tätigkeiten zu erreichen, um anschließend die Ausbalancierung des Alltags von Pflichten und angenehmen Dingen zu erlangen (35). Für die allgemeine Aktivierung spielt auch Sport eine wichtige Rolle. Mithilfe gezielter Bewegungsmaßnahmen lassen sich bestimmte psychische Funktionen wie Konzentration und Motivation verbessern (36). Ein soziales Netz sowie soziale Unterstützung spielen in der Behandlung der psychosozialen und beruflichen Folgen eine wichtige Rolle. Der Betroffene sollte Quellen sozialer Unterstützung und Hilfe identifizieren und Schwachstellen im persönlichen Hilfsnetz erkennen (35).

\section{Depression und (Wie- der-)Einstig ins Berufsleben}

Bei Verlust des Arbeitsplatzes oder längerem Ausbleiben von beruflicher Kontinuität durch Arbeitsunfähigkeit kann sowohl stationär als auch ambulant eine Beratung zur beruflichen Wiedereingliederung und Rehabilitation erfolgen, u. a. in Form von Arbeitgebergesprächen. Hier können die Konkretisierung des beruflichen Wiedereinstiegs, der Umgang mit Anforderungen am Arbeitsplatz, Änderungen des Arbeitsplatzes sowie die berufliche Wiedereingliederung geklärt werden (33). Die rasche Rückkehr ins Arbeitsleben hat therapeutische und sekundärprophylaktische Effekte, da dauerhafte Arbeitsunfähigkeit oder Verrentung der beruflichen und sozialen Teilhabe entgegenstehen. Hier gibt es jedoch oft Defizite, da die Berufs- und Arbeitswelt in der psychiatrisch-psychotherapeutischen oder hausärztlichen Versorgung oft $\mathrm{zu}$ wenig Beachtung findet. Dabei gibt es im Bereich der beruflichen Wiedereingliederung eine Vielzahl von Maßnahmen, von der gestuften Wiedereingliederung („Hamburger Modell'), über das Betriebliche Eingliederungsmanagement (BEM) bis hin zur Umschulung.

In letzter Zeit wurden zudem Modellprojekte entwickelt, die in spezifischen $\mathrm{Si}$ - tuationen präventiv ansetzen sollen, um depressive Erkrankungen im Kontext Arbeit frühzeitig zu erkennen bzw. die Rückkehr an den Arbeitsplatz nach depressiven Episoden möglichst zu erleichtern. Die Entwicklung eines betriebsnahen Versorgungsnetzwerkes stellt eine Möglichkeit der Unterstützung dar. So gibt es erfolgreiche Angebote für psychotherapeutische Sprechstunden im Betrieb: Beschäftigte mit psychischen Problemen können im Betrieb mit einem Arzt und gegebenenfalls einem Psychologen sprechen. Die Sprechstunde umfasst einen Arztbesuch, eine ausführliche Diagnostik, eine Krisenintervention bei Bedarf sowie, falls nötig, eine Überweisung zur weitergehenden Betreuung (37). Eine US-Studie zeigte, dass sich Investitionen in eine verbesserte Versorgung der Arbeitnehmer bei Depressionen auf Unternehmensseite durch niedrigere Fehlzeiten sowie geringere Fluktuation bezahlt machen (38).

Bereits während einer stationären-psychiatrischen Behandlung sollte der berufliche Wiedereinstieg in den Fokus genommen werden. Als mögliche Maßnahme kann das von Hölzle et al. entwickelte Psychoedukationsmodul Rückkehr an den Arbeitsplatz genannt werden (39). Die 5-teilige Intervention bietet gezielte Informationen zu den rechtlichen Hintergründen der beruflichen Wiedereingliederung und Unterstützungsmöglichkeiten im Betrieb sowie Übungen und Austausch zum Umgang mit der Diagnose am Arbeitsplatz. Auch die kognitiv-verhaltenstherapeutische Therapiegruppe hat sich bewährt: In 4 Modulen werden die Themen Berufliches Wohlbefinden/Motivation, Umgang mit Kollegen und Vorgesetzten, Arbeitsbewältigung sowie berufliche (Neu-)Orientierung erarbeitet (40).

Es gilt zudem eine Verbesserung im Übergang von der stationären Behandlung in die ambulante Weiterbetreuung herzustellen. Das vom Innovationsfond des GBA geförderte Forschungsprojekt RETURN stellt sich momentan dieser Aufgabe. Im Rahmen einer randomisierten Studie wird der Frage nachgegangen, ob die Etablierung von Return-to-Work-Experten die Rückkehrchancen an den Arbeitsplatz nach einem stationär-psychiatrischen Aufenthalt verbessern kann. 


\section{Fazit für die Praxis}

Depressive Störungen verursachen schwerwiegende Belastungen, die mit weitreichenden psychosozialen und beruflichen Folgen sowohl für die Betroffenen als auch für ihr soziales Umfeld einhergehen. Trotz des umfangreichen Wissens zu der Schwere der Erkrankung, ist die Versorgung noch nicht optimal. Lediglich 10\% der Betroffenen erhalten eine Leitlinie angemessene ärztliche oder psychotherapeutische Behandlung (2, 22). Auch in Bezug auf die Reintegration in den Arbeitsmarkt ist noch Verbesserungsbedarf vorhanden. Laut Befragungen der Hans-Böckler-Stiftung und des Instituts für Arbeitsmarkt- und Berufsforschung fehlen u. a. geeignete Vermittlungskonzepte sowie flexible Arbeitszeitmodelle (22). Die Versorgung der Patienten sollte mehr nach den tatsächlichen Bedürfnissen der Betroffenen ausgerichtet werden. Es gilt nicht nur eine größere Spezifität im Bereich der Diagnosestellung zu erlangen, sondern diese auch im Bereich der Versorgung zu gewährleisten. Dem hohen Risiko der Chronifizierung dieser Erkrankung kann nur mit einer kontinuierlichen und abgestimmten medizinischen, psychotherapeutischen und psychosozialen Versorgung entgegengewirkt werden (15). Dabei muss neben der besten Akuttherapie auch der Aspekt der Rückkehr in den Beruf bzw. die Klärung beruflicher Perspektive Berücksichtigung finden. Arbeit macht nicht krank, sondern hat in der Regel salutogenetische Wirkungen, die im medizinischen Hilfesystem zu wenig Beachtung finden.

\section{Interessenkonflikt}

Es bestehen keine Interessenkonflikte.

\section{Literatur}

1. Stiftung Deutsche Depressionshilfe. DeutschlandBarometer Depression [Internet]. 06.12.2017 [zitiert am 18.06.2018]. URL: https://www.deutschedepressionshilfe.de/news/details/wie-denktdeutschland-ueber-depression

2. Bassler M, Steffens M. Depression - Familie und Arbeit. Gesellschaftlich und individuelle Auslöser und Auswirkungen einer der häufigsten psychischen Erkrankungen. Frankfurt: Referenz-Verlag 2015.
3. Deutsche Rentenversicherung Bund. Positionspapier der Deutschen Rentenversicherung zur Bedeutung psychischer Erkrankungen in der Rehabilitation und bei Erwerbsminderung. [Internet]. 01.01.2014 [zitiert am 11.06.2018]. URL: https://www.deutsche-rentenversicherung.de/All gemein/de/Inhalt/3_Infos_fuer_Experten/01_sozialmedizin_forschung/downloads/konzepte_systemfragen/positionspapiere/pospap_psych_Erkrankung.html

4. Bramesfeld A, Schwartz F. Volkskrankheit Depression: Bestandsaufnahme und Perspektiven. Psychiatrische Praxis 2007; 34: 247-251.

5. Gesundheitsberichterstattung des Bundes. Heft 51 - Depressive Erkrankungen. Berlin: Robert KochInstitut 2010

6. Busch MA, Maske UE, Ryl L, Schlack R, Hapke U. Prävalenz von depressiver Symptomatik und diagnostizierter Depression bei Erwachsenen in Deutschland. Bundesgesundheitsblatt 2013; 56: 733-739.

7. Jacobi F, Klose M, Wittchen HU. Psychische Störungen in der deutschen Allgemeinbevölkerung: Inanspruchnahme von Gesundheitsleistungen und Ausfalltage. Bundesgesundheitsblatt 2004; 47: 736-744.

8. Spießl H. Depression - unerkannte Volkskrankheit Nr. 1? Nervenheilkunde 2006; 25: 166-169.

9. Ruppe A, Keller F, Wolfersdorfer M. Die Psychosoziale Situation im nachstationären Verlauf Depressiver. Ergebnisse der Weissenauer 6-JahresKatamnese. In: Wolfersdorfer M. Depressionsstationen, Stationäre Depressionsbehandlung: Konzepte, Erfahrungen, Möglichkeiten heutiger Depressionsbehandlung. Berlin: Springer 1997; 236-251.

10. Kühn KU, Quednow BB, Barkow K, Heun R, Linden M, Maier W. Chronifizierung und psychosoziale Behinderung durch depressive Erkrankungen bei Patienten in der Allgemeinarztpraxis im Einjahresverlauf. Der Nervenarzt 2002; 73: 644-650.

11. Ebmeier K, Donaghey C, Steele JD. Recent developments and current controversies in depression. Lancet 2006; 367: 153-167.

12. Dilling H, Freyberger HJ. ICD-10 - Taschenführer zur ICD-10 Klassifikation psychischer Störungen. 8. Aufl. Göttingen: Hogrefe 2015.

13. Judd LL, Akiskal HS, Zeller PJ. Psychosocial disability during the long-term course unipolar major depressive disorder. Arch Gen Psychiatry 2000; 57: 375-380.

14. Schicktanz P. Interpersonale Beziehungsgestaltung und Depression: Eine kulturvergleichende Untersuchung in Chile und Deutschland. [Dissertation]. Heidelberg: Fakultät für Verhaltens- und Empirische Kulturwissenschaften der Ruprecht-KarlsUniversität Heidelberg 2015.

15. DGPPN, BÄK, KBV, AWMF. Nationale VersorgungsLeitlinie Unipolare Depression. [E-Book]. 2. Auflage. Springer 2017.

16. Frei A, Ajdacic-Gross V, Rössler W, Eich-Höchli D. Auswirkungen von depressiven Störungen auf objektive Lebensqualitätsbereiche. Psychiatrische Praxis 2004; 31: 298-303.

17. Lenz A. Depressiv erkrankte Eltern und deren Kinder. In: Bassler M, Steffens M. Depression Familie und Arbeit. Gesellschaftlich und individuelle Auslöser und Auswirkungen einer der häu- figsten psychischen Erkrankungen. Frankfurt: Referenz-Verlag 2015; 87-140.

18. Krug S, Wittchen HU, Lieb R, Beesdo-Baum K, Knappe $S$. Family functioning mediates the association between parental depression and low self-esteem in adolescents. Journal of Affective Disorders 2016; 203: 184-189.

19. Zimmermann U. Der Verlauf der psychosozialen Integration bei der Major Depression unter Berücksichtigung der Episodenzahl. In: Wolfersdorfer M. Depressionsstationen, Stationäre Depressionsbehandlung: Konzepte, Erfahrungen, Möglichkeiten heutiger Depressionsbehandlung. Berlin Heidelberg: Springer 1997; 263-268.

20. Hau S. Psychosoziale Probleme bei Patienten mit chronischer Depression. In: Schlosser A-M, Bernstein E. Grenzen überschreiten - Unterschiede integrieren. Bonn: Psychosozial Verlag 2012; 255-273.

21. Unger HP. Depression und Arbeitswelt. Psychiatrische Praxis 2007; 34: 256-260.

22. BPtK - BundesPsychotherapeutenKammer. BPtKStudie zur Arbeits- und Erwerbsunfähigkeit - Psychische Erkrankungen und gesundheitsbedingte Frühverrentung. [Internet]. 2013. [zitiert am 18.06.2018]. URL: https://www.bptk.de/uploads/ media/20140128_BPtK-Studie_zur_Arbeitsund_Erwerbsunfaehigkeit_2013_1.pdf

23. Kessler RC, Akiskal HS, Ames M, Birnbaum H, Greenberg P, Hirschfield RM, Jin R, Merikangas KR, Simion GE, Wang PS. Prevalence and effects of mood disorders on work performance in a nationally representative sample of U.S. Workers. Am J Psychiatry 2006; 163: 1561-1568.

24. Jacobi F. Nehmen psychische Störungen zu? Reportpsychologie 2009; 34: 16-28.

25. Jacobi F. Der Hype um die kranke Seele. Gesundheit und Gesellschaft 2012; 5: 23-27.

26. Badura, Ducko, Schröder, Klose, Meyer. Fehlzeiten-Report 2017: Krise und Gesundheit Ursachen, Prävention, Bewältigung. Berlin: Springer 2017.

27. Rau R, Henkel D. Zusammenhang von Arbeitsbelastungen und psychischen Erkrankungen - Review der Datenlage. Nervenarzt 2013; 84: 791-798.

28. DAK Gesundheit. Gesundheitsreport 2018 [Internet]. März 2018 [zitiert am 15.06.2018]. URL: https://www.dak.de/dak/bundes-themen/gesund heitsreport-2018-1970320.html

29. Schmacke N. Häufigkeit seelischer Erkrankungen. Die Frage nach der "wahren" Prävalenz ist kein akademischer Luxus. GGW 2012; 3: 7-15.

30. Klinker K, Rennert D, Richter M. Gesundheit in Regionen - Blickpunkt Psyche. BKK Gesundheitsatlas 2015. Berlin: MWV Medizinisch Wissenschaftliche Verlagsgesellschaft 2015.

31. Siegrist J, Dragano N. Psychosoziale Belastungen und Erkrankungsrisiken im Erwerbsleben. Befunde aus internationalen Studien zum Anforderungs-Kontroll-Modell und zum Modell beruflicher Gratifikationskrisen. Bundesgesundheitsblatt 2008; 51: 305-312.

32. Ehrenberg A. Das erschöpfte Selbst. Depression und Gesellschaft in der Gegenwart. Frankfurt/ Main: Campus Verlag 2004.

33. Konrad C. Therapie der Depression. Praxisbuch der Behandlungsmethoden. [E-Book]. Berlin Heidelberg: Springer-Verlag 2017. DOI: 10.1007/9783-662-50347-8. 
34. Schaub A, Roth E, Goldmann U. Kognitiv-psychoedukative Therapie zur Bewältigung von Depressionen. Ein Therapiemanual. [E-Book]. 2. Aufl. Göttingen: Hogrefe Verlag 2013.

35. Hautzinger M. Kognitive Verhaltenstherapie bei Depressionen. [E-Book]. 7. Aufl. Basel: Beltz Verlag 2013.

36. DGPPN - Deutsche Gesellschaft für Psychiatrie, Psychotherapie und Nervenheilkunde. S3-Leitlinie Psychosoziale Therapien bei schweren psychischen Erkrankungen. S3-Praxisleitlinien in
Psychiatrie und Psychotherapie.[E-Book]. Berlin Heidelberg: Springer-Verlag 2013.

37. Rothermund E, Kilian R, Rottler E, Mayer D, Hölzer M, Rieger MA, Gündel H. Improving access to mental health care by delivering psychotherapeutic care in the workplace: A cross sectional exploratory trial. PLOS ONE 2017; 12(1): e0169559.

38. Wang PS, Patrick A, Avorn J. The costs and benefits of enhanced depression Care to employers. Arch Gen Psychiatry 2006; 63: 1345-1353.
39. Hölzle P, Baumbach A, Mernyi L, Hamann J. Psychoedukationsmodul Rückkehr an den Arbeitsplatz - Eine Interventionsstudie. Psychiat Praxis 2017, DOI: 10.1055/s-0043-105775.

40. Koch S, Hedlund S, Rosenthal S, Hillert A. Stressbewältigung am Arbeitsplatz: Ein stationäres Gruppentherapieprogramm. Verhaltenstherapie 2006; 16: 7-15. 


\section{Zertifizierte Fortbildung}

\section{Fragen zum Thema „Psychosoziale und berufliche Folgen der Depression"}

1. Wie hoch liegt die Lebenszeitprävalenz für Depressionen in der Allgemeinbevölkerung?
a) $9 \%$
b) $11 \%$
c) $19 \%$
d) $23 \%$
e) $27 \%$

2. Die Messgröße YLD (years of life with disability) erfasst die mit Behinderung gelebten Lebensjahre. Wieviel \% der weltweit erlebten YLD sind durch Depressionen verursacht?
a) $11,8 \%$
b) $15,7 \%$
c) $19,2 \%$
d) $21,6 \%$
e) $38,3 \%$

\section{Was ist nicht ein Hauptsymptom} der Depression nach ICD-10?

a) Gedrückte Stimmung.

b) Interessenverlust.

c) Eingeschränkte Konzentrationsfähigkeit.

d) Verminderter Antrieb.

e) Freudlosigkeit.

\section{Kinder depressiver Eltern sind häufig} selbst gefährdet, an einer depressiven Störung zu erkranken. Um das Wievielfache ist das Risiko der eigenen Erkrankung bei betroffenen Kindern erhöht?

a) Nicht erhöht.

b) Zweifache.

c) Dreifache.

d) Fünffache.

e) Sechsfache.

5. Was versteht man unter dem Begriff Parentifizierung?

a) Unter Parentifizierung versteht man die Umkehr der sozialen Rollen, bei der das Kind die Elternfunktion übernimmt.

b) Unter Parentifizierung versteht man die erlebten Schwierigkeiten depressiver Eltern im Umgang mit ihren Kindern.

c) Unter Parentifizierung versteht man die erhöhten Anforderungen, die an den nicht erkrankten Elternteil gestellt werden. d) Unter Parentifizierung versteht man das gesteigerte Risiko eines Kindes erkrankter Eltern, selbst zu erkranken.

e) Unter Parentifizierung versteht man die adäquate Übernahme der Elternrolle trotz psychischer Erkrankung.

6. Depressiv Erkrankte zeigen u. a. eine herabgesetzte Leistungsfähigkeit. Wie viele Abwesenheitstage pro Monat entspricht die reduzierte Arbeitsleistung nicht krankgeschriebener Depressiver?

a) 1,8 .

b) 2,3 .

c) 3,4 .

d) 5 .

e) 6,7 .

7. Welche der folgenden Aussagen ist keine Erklärung für die gestiegenen Arbeitsunfähigkeits- sowie Frühverrentungszahlen aufgrund psychischer Störungen?

a) Die "Normalisierung" psychischer Störungen führt zu einer erhöhten Aufmerksamkeit psychischer Probleme und deren Symptome.

b) In den letzten Dekaden kam es zu einer bedeutsamen Erhöhung der allgemeinen Prävalenz psychischer Störungen.

c) Psychische Störungen werden von Hausärzten häufiger erkannt als früher.

d) Veränderungen und zunehmende Belastungen im Arbeitsleben führen zu einem $\mathrm{Zu}$ wachs psychischer Störungen.

e) Aufgrund des Zusammenhangs zwischen somatischen Erkrankungen und Depression entsteht häufig ein Diagnosewechsel hin zur psychischen Erkrankung.

8. Psychoedukation stellt eine Grundvoraussetzung der Depressionsbehandlung dar. Was steht bei dieser Intervention nicht im Vordergrund?

a) Förderung der Eigenverantwortung des Patienten.

b) Besseres Verständnis der Symptome.

c) Entlastung der Angehörigen.

d) Vermittlung von Informationen zu Behandlungsoptionen.

e) Abbau von Schuldgefühlen bei Angehörigen.
CME-Fortbildung online

Die Teilnahme an dieser durch die Ärztekammer Nordrhein zertifizierten CME-Fortbildung ist für 12 Monate ausschließlich online möglich. Zur Anmeldung gehen Sie bitte auf http://cme.thieme.de. Dort erfahren Sie auch den genauen Teilnahmeschluss. Es ist immer nur eine Antwort pro Frage zutreffend. Als Leser der Nervenheilkunde nehmen Sie kostenlos am CME-Programm teil. Je nach CME-Fortbildung erhalten Sie bis zu vier Fortbildungspunkte. Weitere Informationen zur Anmeldung und Registrierung finden Sie unter http://cme.thie me.de. Sie erhalten bei inhaltlichen und technischen Fragen tutoriellen Support. VNR 2760512018154652937

9. Eine Methode der Förderung von Aktivitäten in der Behandlung Depressiver ist die Verhaltensaktivierung. Worum geht es hierbei?

a) Bei der Verhaltensaktivierung geht es um die Befähigung der Patienten, eigene Bedürfnisse durchsetzen zu können.

b) Bei der Verhaltensaktivierung geht es darum, mittels gezielter Bewegungsmaßnahmen bestimmte psychische Funktionen zu verbessern.

c) Bei der Verhaltensaktivierung geht es um das Erreichen eines angemessenen Aktivitätsniveaus mit einer ausgewogenen Tagesstruktur.

d) Bei der Verhaltensaktivierung geht es ausschließlich um die Bewältigung der alltäglichen Pflichten.

e) Bei der Verhaltensaktivierung geht es um die Vermittlung sozialer Kompetenzen.

10. Welche Aussage zum Wiedereinstieg ins Berufsleben ist richtig?

a) Die Berufs- und Arbeitswelt ist in der psychiatrisch-psychotherapeutischen Versorgung gut verankert.

b) Es gibt bislang nur wenige Maßnahmen zur Förderung des Wiedereinstiegs ins Berufsleben.

c) Die Rückkehr ins Arbeitsleben hat therapeutische und sekundärprophylaktische Effekte.

d) Der berufliche Wiedereinstieg sollte erst nach einer stationär-psychiatrischen Behandlung in den Fokus genommen werden.

e) Das „Hamburger Modell“ ist eine alternative Bezeichnung für das Betriebliche Eingliederungsmanagement. 